\title{
VLA Dashboard: Um Mecanismo para Visualização do Desempenho de Estudantes de Matemática no Ensino Médio
}

\author{
Euler Vieira da Silva - PPGI - IComp - UFAM / IFAM Campus Maués \\ eulervieira@ifam.edu.br \\ José Francisco de Magalhães Netto - PPGECIM/PPGI - IComp - UFAM \\ jnetto@icomp.ufam.edu.br \\ Ricardo Augusto Lima de Souza - PPGECIM - ICE - UFAM / UFAM Coari \\ ricardo_souza@ufam.edu.br
}

\begin{abstract}
Resumo. Este artigo descreve as contribuições do uso de um mecanismo VLA Dashboard para visualização do desempenho de estudantes do Ensino Médio em avaliações de Matemática Básica aplicadas no Moodle. Diante disso, empregou-se uma pesquisa qualitativa do tipo Estudo de Caso. Em seguida, a coleta foi aplicada em três etapas: pré-Teste, oficina de capacitação e aplicação de avaliação diagnóstica pelos professores. Também, foi usado o Modelo de Aceitação de Tecnologia para os questionários. Os resultados apontam que o uso das técnicas de Visualização da Informação, learning analytics e abordagem multiagente contribuem para melhorar os diagnósticos das atividades avaliativas do professor.
\end{abstract}

Palavras-chave: avaliação em matemática, dashboard, learning analytics, sistemas multiagente, visualização.

\begin{abstract}
This article describes the contributions of the use of a mechanism for visualization of the performance of students of high school in assessments of Basic Mathematics applied in Moodle. In addition, was used a qualitative research of the Case Study type. Then, the collection was applied in three stages: pre-test, training workshop and application of diagnostic evaluation by teachers. Also, the Technology Acceptance Model was used for the questionnaires. The results show that the use of Information Visualization techniques, learning analytics and multiagent approach contribute to improve the diagnoses of the teacher's evaluation activities.
\end{abstract}

Keywords: assessment in mathematics, dashboard, learning analytics, multiagent systems, visualization.

\section{Introdução}

Atualmente nas escolas públicas de Ensino Médio, torna-se cada vez mais difícil para o professor identificar as dificuldades individuais ou coletivas em conteúdos específicos da disciplina de Matemática, tendo em vista fatores, tais como: carga horária elevada, estudantes desmotivados ou com dificuldades de aprendizagem. Muitas vezes, esta identificação só é percebida por meio do resultado da nota final do estudante das atividades letivas que compõem o processo educacional (bimestre, semestre ou ano).

Conforme o relatório apresentado pelo Instituto Nacional de Estudos e Pesquisas Educacionais Anísio Teixeira (INEP), órgão vinculado ao Ministério da Educação (MEC), o nível de aprendizado dos estudantes brasileiros no Ensino Médio piorou em Matemática e chegou, em 2015, ao pior resultado desde 2005, início de uma série histórica do Sistema de Avaliação da Educação Básica (SAEB), também conhecida como Prova Brasil, este documento registra as médias dos estudantes nesta disciplina ao longo de vinte anos (INEP, 2016). 
Nesse contexto é fundamental que os desempenhos dos estudantes sejam identificados pelos professores a tempo de agir com intervenções pedagógicas e motivacionais, tornam-se necessárias ferramentas que forneçam essas informações dinamicamente durante suas atividades, pois possibilita aos professores, visualizar essas informações de forma rápida e simplificada (ARANTES; SEABRA, 2016).

Diante desse cenário, enuncia-se a seguinte questão de pesquisa: Quais são as contribuições do uso de um mecanismo para visualização do desempenho de estudantes do $1^{\circ}$ Ano do Ensino Médio em avaliações de Matemática Básica pelo professor?

Igualmente importante, o objetivo geral desta pesquisa é descrever as contribuições do uso de um mecanismo (VLA Dashboard) para visualização do desempenho de estudantes do $1^{\circ}$ Ano do Ensino Médio, em avaliações de Matemática Básica pelo professor aplicadas no Ambiente Virtual de Aprendizagem Moodle.

Além desta introdução, o artigo está estruturado da seguinte forma: a Seção 2 apresenta a Fundamentação Teórica; a Seção 3 os Trabalhos Relacionados; a Seção 4 a VLA Dashboard; na Seção 5 o Metodologia; a Seção 6 exibe os Resultados e Discussões; e por fim, na Seção 7 são feitas as Considerações Finais.

\section{Fundamentação Teórica}

Esta seção apresenta uma breve descrição dos conceitos importantes para o entendimento da pesquisa, tais como: Learning Analytics, Dashboards de Aprendizagem e Sistemas Multiagente.

\subsection{Learning Analytics (LA)}

A Learning Analytics é uma área de pesquisa que surgiu como uma consequência do crescente número de plataformas educacionais on-line e a necessidade de entender como a aprendizagem mediada por tecnologia acontece. Assim, LA está relacionada à coleta de dados, na qual são analisados os dados produzidos por estudantes dentro e fora dos AVAs, com o propósito de melhorar o processo de ensino-aprendizagem.

Com efeito, Siemens (2011) define LA como a medida, coleta, análise e relato dos dados de estudantes e seus contextos com o propósito de entender e potencializar o aprendizado no ambiente em que ocorre. De forma similar, Johnson, Adams e Cummins (2012) apresentam no relatório da NMC Horizon Report definição mais abrangente, referindo-se a LA como à interpretação de uma ampla gama de dados produzidos e recolhidos em nome dos estudantes, a fim de avaliar o progresso acadêmico, prever o desempenho futuro e detectar possíveis problemas.

Apesar das diferentes definições, é possível afirmar que todas elas têm ênfase na compreensão de dados educacionais para o sucesso na aprendizagem. Neste trabalho, LA é entendida como uma área da análise de dados que coleta e processa dados sobre estudantes, a fim de gerar conhecimento para auxiliar o professor no seu cotidiano escolar.

Desta forma, LA quando aplicada para análise automática de dados e interpretada corretamente, permite ao professor entender com mais exatidão as necessidades de aprendizagem dos estudantes e adequar a instrução de forma apropriada e com maior precisão, uma vez que LA tem implicações não apenas para o desempenho individual dos estudantes, mas também como os educadores percebem os processos de ensino, aprendizado e avaliação.

\subsection{Dashboards de Aprendizagem}

O Dashboard é definido como uma exibição visual da informação importante e necessária para alcançar um ou mais objetivos, os quais consolidados e organizados em 
tela única podem ser monitorados rapidamente. São representados em gráfícos interativos, onde cada um apresenta uma visão diferente de um conjunto de dados comuns (FEW, 2004, 2006).

Logo, o uso de Dashboards proporcionam aos seus usuários, analisar os dados de diferentes perspectivas e em vários níveis, uma vez que é um meio de comunicação que reúne métricas em uma única visão consolidada. Por exemplo, na educação, o emprego de Dashboards simplifica a visualização e o entendimento das informações geradas por estudantes em AVAs, podendo ser facilmente analisadas, contribuindo ao professor, apoio na tomada de decisões.

Similarmente, Duval (2011) afirma que Dashboard de Aprendizagem pode ser extremamente útil para estudantes e professores, pois exibe uma visão geral de suas atividades e de como eles se relacionam. Para Charleer et al. (2014) Dashboards de Aprendizagem consistem em gráficos que ajudam o usuário a explorar grande quantidade de dados gerados de forma interativa, exibindo uma visão geral combinada com informações úteis a professores e estudantes na tomada de decisão.

Segundo Park e Jo (2015), atualmente os Dashboards de Aprendizagem são divididos em três tipos: o primeiro, Dashboard para Professores, geralmente informam sobre o estado da aprendizagem do estudante, fornecendo feedback, avaliação e classificação, além de ajudar a desempenhar seu papel de forma eficaz; o segundo, Dashboard para Estudantes, apresentam padrões aos estudantes, ajudando-os a modificar suas estratégias, motivando-os a aprendizagem e a autorreflexão; o terceiro, Dashboards para apoio a Professores e Estudantes, que reúnem características comuns aos Dashboards anteriores.

\subsection{Sistemas Multiagente (SMA)}

Os autores Russell e Norvig (2013) definem Agente Inteligente (AI) como uma entidade autônoma, capaz de perceber seu ambiente por meio de sensores e de agir sobre esse ambiente por intermédio de atuadores. Do mesmo modo, Wooldridge (2009) registra que agentes são sistemas computacionais capazes de ações autônomas em algum ambiente, com a finalidade de alcançar seus objetivos de projeto e devem apresentar as seguintes características básicas:

- Autonomia: capacidade de executar ações sem intervenção direta de seres humanos ou de outro sistema;

- Habilidade Social: capacidade de comunicar com seres humanos ou com outros agentes, por meio de troca de mensagens para realizar tarefas.

- Reatividade: capacidade de perceber mudanças no seu ambiente e responder em tempo hábil às ações que ocorrem no ambiente;

- Proatividade: capacidade de exibir um comportamento que prioriza agir a cumprir metas definidas, tomando iniciativa quando necessário, para satisfazer seus objetivos.

Tais características são importantes para o desempenho do Sistema de Informação que usa AIs ou um SMA. De acordo com Jaques e Oliveira (2000), um SMA é um sistema formado por mais de um agente, construindo desta forma, um Sistema Multiagente.

Assim, em função da complexidade desse trabalho, sua natureza distribuída e relativa semelhança com problemas do mesmo tipo já solucionados, será aplicada uma abordagem multiagente, como parte da solução para este problema, a fim de coletar, analisar e notificar dinamicamente informações pré-definidas pelo mecanismo.

\section{Trabalhos Relacionados}

O trabalho de Schwendimann (2016) registra uma Revisão Sistemática da Literatura 
(RSL) aplicada no período de (2010-2015) sobre o Estado da Arte em Dashboards de Aprendizagem nos campos de Learning Analytics e Education Data Mining. O objetivo da RSL foi identificar quais dados são significativos para as diferentes partes interessadas (professores, estudantes, administradores e pesquisadores) e como esses dados podem ser apresentados para apoiar processos de sensemaking. Os resultados apontam que $53 \%$ dos trabalhos publicados atualmente são focados em ambientes universitários e destacam a necessidade de estudos de Dashboard em outros ambientes, como por exemplo, no K-12 ${ }^{1}$.

No trabalho de Kamin e Capitanu (2008) é descrito um dos estudos pioneiros sobre o uso de Dashboards para apoio ao professor, o NuPaper Dashboard, que tem o objetivo de empregar o monitoramento das atividades dos estudantes de uma turma de Álgebra do Ensino Médio. Por conseguinte, os estudantes usam os Tablets como "lápis e papel" para responder a exercícios da disciplina individualmente ou em grupo no aplicativo empregado em sala de aula. Para cada uma das aulas, o professor prepara os estudantes com os conteúdos da disciplina, e em seguida passam o restante do tempo respondendo os exercícios no Tablet. Os autores registram que o NuPaper Dashboard facilita o monitoramento das informações dos estudantes na resolução de exercícios, porém precisa de aperfeiçoamentos para ser aplicado em um número maior de turmas.

Na mesma direção, Podgorelec e Kuhar (2011) descrevem o Moodle Dashboard, uma ferramenta que tem o propósito de exibir informações de desempenho e hábitos de acesso dos estudantes para apoio a professores universitários a partir de dados coletados no Moodle. Acrescente-se que, no Moodle Dashboard são exibidos ao professor informações referentes as frequências e as notas dos estudantes, bem como da evolução dos trabalhos submetidos em gráficos de linha e box plot.

Por fim, Einhard, Tavares e Cechinel (2016) descrevem o Moodle Analytics Dashboard (MAD), uma ferramenta de LA desenvolvida como extensão para o navegador Google Chrome ou derivados, de funcionalidade local para diferentes plataformas desktops. O MAD possibilita aos seus usuários a visualização dos registos de acesso $(\log s)$ de estudantes e professores em disciplinas oferecidas no Moodle, com o objetivo de auxiliar o professor no acompanhamento do seu processo de ensinoaprendizagem, além de ajudar na identificação de estudantes em risco.

A principal contribuição do VLA Dashboard em relação às abordagens anteriormente apresentadas é fornecer informações detalhadas sobre as dificuldades dos estudantes em conteúdos específicos da disciplina ao professor. O mecanismo será exposto e discutido na Seção 4.

\section{VLA Dashboard}

O VLA Dashboard é um mecanismo desenvolvido para a plataforma Web com o objetivo de fornecer aos professores a visualização dinâmica do desempenho de estudantes em avaliações realizadas em AVAs. A abordagem do VLA Dashboard consiste em contribuir na identificação de estudantes com dificuldades em conteúdos específicos de cursos ou disciplinas, incentivando o professor na prática de reflexão sobre o nível de aprendizagem da turma ou um estudante específico.

Para estruturar o banco de questões no Moodle e gerar dados para o VLA Dashboard, foi utilizada a Matriz de Referência de Matemática da $9^{\circ}$ Ano do Ensino Fundamental disponível no Plano de Desenvolvimento da Educação (PDE) de 2008 (BRASIL, 2008).

Desta forma, para cada questão pertencente a um Descritor, no momento da

${ }^{1} \mathrm{~K}-12$ é uma denominação para educação primária e a educação secundária como um todo. É usada nos Estados Unidos, em algumas partes da Austrália, Canadá e Índia. 
inserção do banco de questões, pode ser definida pelo professor em três níveis de dificuldades: fácil, médio ou difícil, os quais auxiliam no momento de elaborar a avaliação e também são usados para gerar gráficos com maior nível de detalhes. $\mathrm{O}$ uso de Temas e Descritores contribuiu para o professor obter resultados mais precisos dos avanços e dificuldades.

$\mathrm{Na}$ implementação do VLA Dashboard foram empregadas ferramentas open source disponíveis na Internet, com destaque para o servidor Apache, a linguagem PHP e o template para desenvolvimento de aplicações Web AdminLTE HTML52, além do framework JADE usado para a criação dos agentes (BELLIFEMINE et al. 2007).

\subsection{Arquitetura do Sistema}

A arquitetura do VLA Dashboard é apresentada na Figura 1 e mostra a visão geral do sistema dividido em três partes fundamentais:

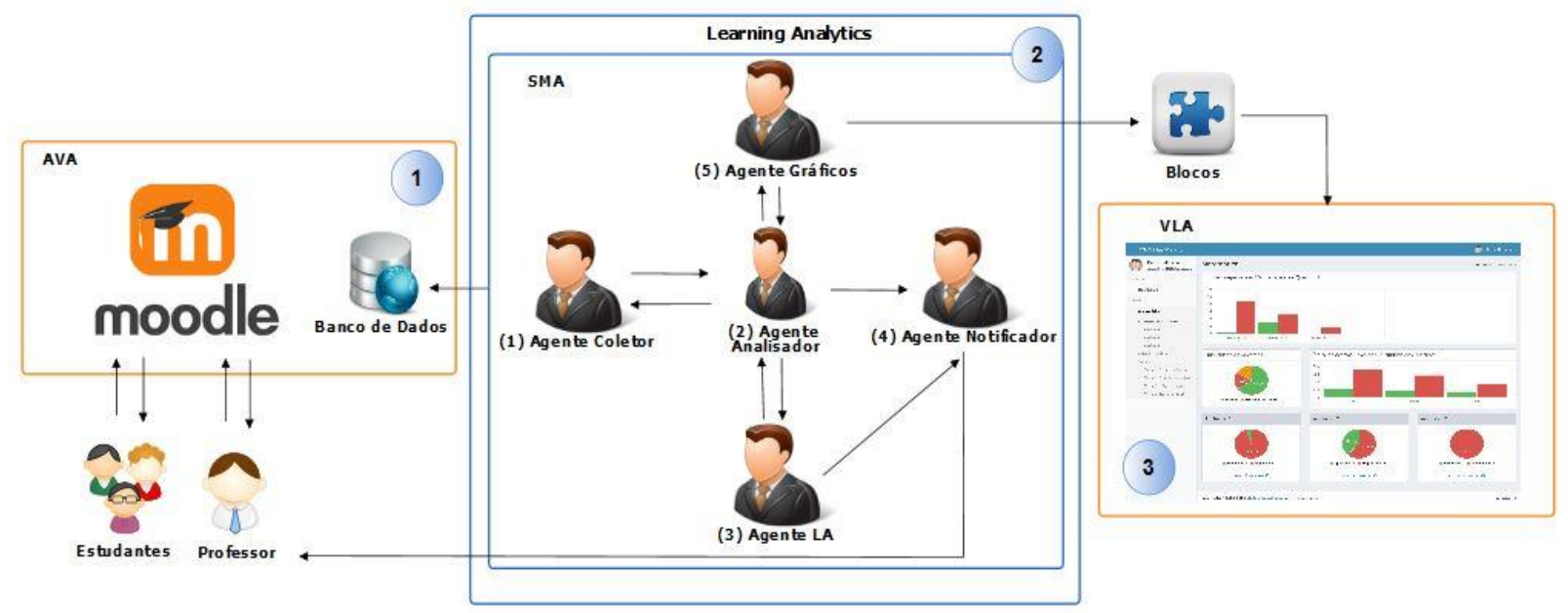

Figura 1 - Visão Arquitetural do Sistema.

1. O AVA, que para exemplo de aplicação, foi usado o Moodle, no qual interagem dois tipos de usuários: professores e estudantes. Os professores têm a possibilidade de inserir questões em um banco de questões e realizar avaliações. Por sua vez os estudantes, respondem as avaliações disponibilizadas no Moodle.

2. O SMA, responsável pela integração do banco de dados do Moodle com o VLA Dashboard é composto por cinco agentes, cada um com seus respectivos papéis. O primeiro (1), Agente Coletor, responsável em coletar diretamente das tabelas dos bancos de dados do Moodle, os dados referentes as notas nas avaliações dos estudantes e da turma, enviando-as diretamente ao Agente (2). O segundo (2), Agente Analisador, tem como o objetivo receber e formatar os dados fornecidos pelo Agente (1), possibilitando que os Agentes (3) e (4) interpretem as informações com as notas das avaliações, de acordo com cada descritor e o nível de dificuldade da questão. O terceiro, Agente LA, Faz o acompanhamento do progresso dos estudantes e da turma, tendo como base o desempenho nas avaliações, de acordo com os descritores das questões fornecidos pelo Agente (2), além de notificar dinamicamente os resultados ao professor por intermédio do Agente (5). O quarto (4), Agente Gráficos, responsável por fornecer gráficos interativos dinamicamente ao professor. Cada gráfico, apresenta uma visão diferente de um conjunto de dados comuns das notas dos estudantes, com as informações analisadas pelo Agente (2). Por fim, o quinto (5), Agente Notificador, que tem a função de notificar automaticamente via e-mail ao 
professor, informações referentes ao desempenho dos estudantes e da turma com base nos dados fornecidos pelos Agentes (2) e (3).

3. O VLA Dashboard, desenvolvido para visualização dinâmica e interativa dos resultados das avaliações realizadas no Moodle. O mecanismo pode ser acessado de duas maneiras. A primeira, por meio de um plugin no Moodle, que ao ser clicado abre uma página $W e b$ em uma nova guia no navegador. A segunda, digitando diretamente o endereço no navegador $W e b$, onde o professor deve informar o nome de usuário e senha para efetuar o login na página3. A Figura 2 ilustra a visão geral do resultado de uma avaliação para o professor.

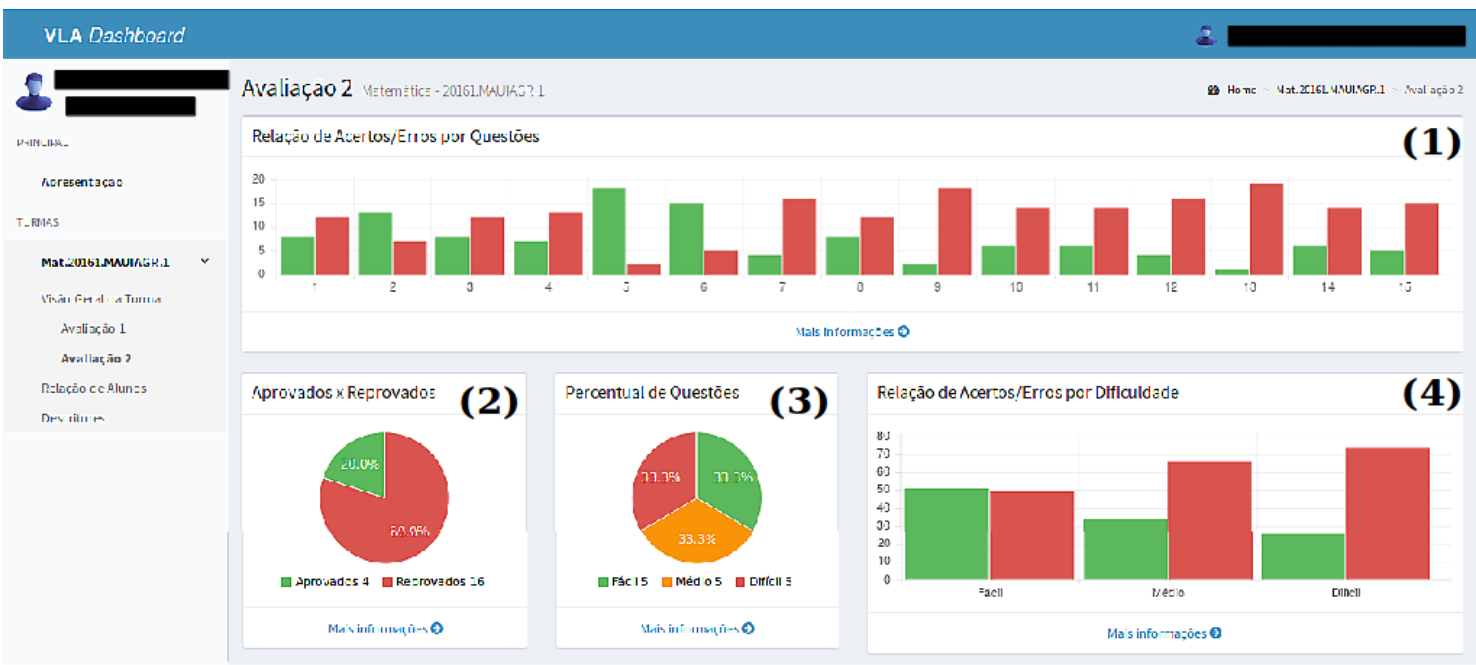

Figura 2 - Visão Geral por Avaliação de uma turma.

Deve-se notar que a avaliação é composta por 26 questões e que as informações são disponibilizadas ao professor em gráficos de barras e setores divididos em quatro blocos. O primeiro (1), Relação de Acertos/Erros por Questões, fornece ao professor a quantidade de erros e acertos por questão da avaliação. $O$ segundo (2), Aprovados/Reprovados, exibe o número total e percentual de estudantes aprovados e reprovados. O terceiro (3), Porcentagem de Questões por Nível de Dificuldade, apresenta o percentual e a quantidade do total de questões realizadas pela turma, agrupados por níveis de dificuldade (fácil, médio e difícil). Por conseguinte, o quarto (4), Relação de Acertos/Erros por Nível de Dificuldade, em que é mostrada a quantidade total de acertos e erros da avaliação agrupada por níveis de dificuldade.

O VLA Dashboard também tem a opção de gerar relatórios detalhados por avaliação que identificam por exemplo, o Tema e o Descritor; a maior nota, a menor nota e a média individual ou da turma relacionados a questão, além de exibir o percentual de erros e acertos assinalados, conforme ilustrado na Figura

Para melhor compreensão, na Figura 3 temos as informações organizadas por seção, cada uma correspondente a questões distintas. Na parte superior da seção, estão identificados o número da questão e o nível dificuldade. Para uma melhor percepção, foram empregadas não apenas as cores verde, amarelo e vermelho indicando o nível de dificuldade fácil, médio e difícil respectivamente, como também sua descrição. 


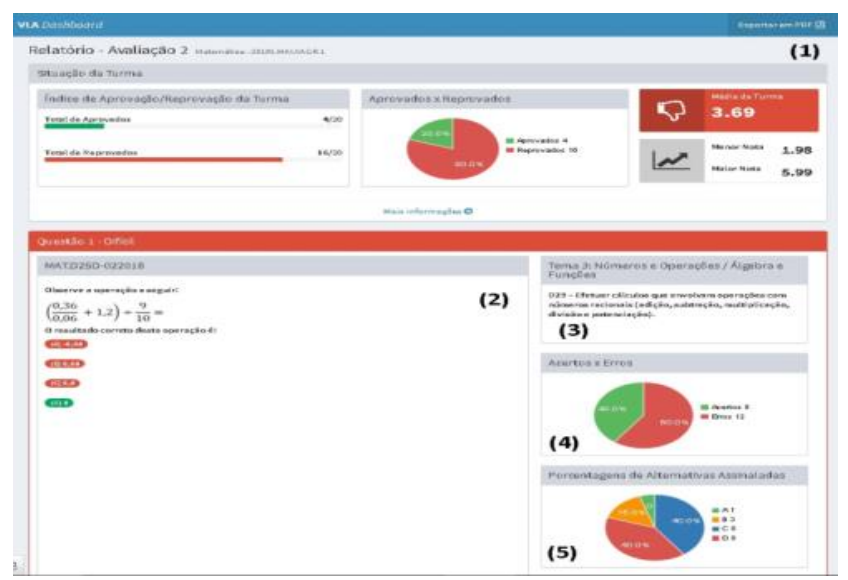

Figura 3 - Relatório por Avaliação de uma turma.

Cada seção está dividida em duas colunas e possui cinco blocos. O primeiro bloco (1): concede informações sobre o nome da avaliação e disciplina, o índice de aprovados / reprovados, a média da turma, a maior e a menor nota, bem como o relatório em PDF da avaliação para download. O segundo bloco (2): identifica não só a sequência e o nível dificuldade da questão, como também o enunciado com as alternativas. $\mathrm{O}$ terceiro bloco (3): mostra o Tema e o Descritor referente a questão. $\mathrm{O}$ quarto bloco (4): fornece o percentual e a quantidade de erros e acertos apontados na questão. O quinto bloco (5): exibe a quantidade e porcentagem por alternativas assinaladas na questão.

O VLA Dashboard combina, LA com SMA, para coletar, analisar e exibir dados dinamicamente por meio de gráficos. Os dados apresentados são filtrados e organizados para facilitar a compreensão dos gráficos gerados, uma vez que diferentes tipos de relatórios foram criados a fim de entregar fornecer ao professor o acompanhamento do desempenho dos estudantes.

Os relatórios anteriormente citados não são a abordagem única e final para acompanhar o desempenho do estudante, porém fornecem detalhes suficientes de cada uma das avaliações, para que o professor possa melhor, avaliar e fornecer uma aprendizagem eficaz e adaptável. Também foi elaborado um relatório por e-mail para facilitar e automatizar o acesso a cada relatório, desempenhado pelo Agente Notificador.

\section{Metodologia}

Este artigo relata uma pesquisa aplicada, descritiva, com uma abordagem qualitativa, delineada por Estudo de Caso para coleta e análise de dados. Segundo Yin (2015), o Estudo de Caso investiga um fenômeno contemporâneo (o "caso") em seu contexto no mundo real. De acordo com Gil (2010), as etapas do Estudo de Caso não se dão numa sequência rígida. Saliente-se ainda que esta pesquisa consiste numa série de procedimentos típicos de pesquisa desta natureza, são eles:

1. Local e Sujeitos da Pesquisa: a pesquisa foi realizada com professores de Matemática do $1^{\mathrm{o}}$ Ano do Ensino Médio de um Instituto Federal de Educação (IFE).

2. Instrumentos de Coleta de Dados: como instrumento para coleta dos dados foram utilizados questionários on-line elaborados no Google Formulários ${ }^{4}$, por permitir a criação e disponibilização gratuita de formulários personalizados. 
3. Procedimentos para Coleta de Dados: a coleta de dados foi desenvolvida em três etapas: Pré-Teste, Oficina de Capacitação e Aplicação de Avaliação Diagnóstica pelos Professores.

4. Validação da Proposta: para validação, foi aplicado o Modelo de Aceitação de Tecnologia, do inglês Technology Acceptance Model (TAM), usado para validação de sistemas da informação (DAVIS, 1989). Esse modelo origina-se da Teoria da Ação Raciocinada, do inglês Theory of Reasoned Action (TRA), que é uma teoria da Psicologia que busca explicar o comportamento dos usuários diante de uma situação específica (FISHBEIN; AJZEN, 1975).

\section{Resultados e Discussões}

O trabalho foi desenvolvido em três etapas: a primeira, Pré-Teste, realizada com um professor e uma turma com a finalidade de identificar a viabilidade do VLA Dashboard. A segunda, uma Oficina de Capacitação para Professores, com o objetivo de mostrar aos usuários como elaborar questionários e avaliações no Moodle, bem como visualizar os resultados destas no Dashboard proposto. Colaboraram para esta etapa três professores e três turmas. Por fim, a terceira, Aplicação de Avaliação Diagnóstica pelos Professores, esta etapa teve o propósito de fornecer informações aos usuários sobre as habilidades dos estudantes em conteúdos específicos já abordados na disciplina de Matemática, por intermédio do VLA Dashboard. Participaram desta etapa, três professores e alunos de quatro turmas. Para examimar a aceitação do VLA Dashboard, empregou-se o modelo TAM, que tenta explicar atitudes e comportamentos dos usuários na aceitação de tecnologias (DAVIS, 1989; MARINHO et al., 2014). O questionário foi organizado com perguntas sobre os dados de identificação, utilidade percebida e facilidade de uso, intenções de uso futuro, compatibilidade com o trabalho, bem como questões de discussão para registro das opiniões sobre as vantagens e desvantagens da abordagem. As subseções a seguir descrevem os quatro construtos que compõem o modelo TAM e também uma questão aberta para comentários dos professores.

\subsection{Utilidade Percebida}

No que se referem às respostas sobre a Utilidade Percebida relativa à questão "utilizar o $V L A$ Dashboard no meu trabalho me permitirá realizar tarefas mais rapidamente", $33,3 \%$ dos respondentes registraram que concordaram, enquanto $66,7 \%$ concordaram totalmente. Sobre a mesma percepção de utilidade para à questão "utilizar o $V L \boldsymbol{A}$ Dashboard iria melhorar o meu desempenho no trabalho", 33,3\% concordam enquanto $66,7 \%$ concordaram totalmente. Igualmente para à questão "utilizar o $\boldsymbol{V L} \boldsymbol{A}$ Dashboard no meu trabalho iria aumentar a minha produtividade", 33,3\% dos respondentes concordam enquanto $66,7 \%$ concordaram totalmente. Em relação às respostas para à questão "utilizar o $V \boldsymbol{L A}$ Dashboard iria aumentar minha eficácia no trabalho", 33,3\% foram indiferentes, enquanto $66,7 \%$ dos respondentes concordam. Quanto às respostas relacionadas à questão "utilizar o VLA Dashboard tornaria mais fácil fazer o meu trabalho", 33,3\% foram indiferentes, 33,3\% concordam e 33,3\% dos respondentes concordaram totalmente. Respondendo o questionamento sobre "eu acharia o VLA Dashboard útil no meu trabalho", 33,3\% dos respondentes concordam enquanto $66,7 \%$ concordaram totalmente. Deve-se perceber que os resultados obtidos sobre o construto de Utilidade Percebida, indicam que utilidade do VLA Dashboard foi avaliada de forma satisfatória pela maioria dos professores participantes, não havendo discordância de sua utilidade nas questões por nenhum dos respondentes. 


\subsection{Facilidade de Uso Percebida}

Quanto às respostas relacionadas à Facilidade de Uso referente à questão "aprender a usar o VLA Dashboard foi fácil pra mim", 66,7\% dos respondentes registraram que concordaram, enquanto 33,3\% concordaram totalmente. Igualmente às respostas para a questão "eu consideraria fácil de controlar o $\mathbf{V L A}$ Dashboard para fazer o que eu quero que ele faça", $66,7 \%$ dos respondentes concordam, enquanto $33,3 \%$ concordaram totalmente. Sobre a questão "minha interação com o $\boldsymbol{V L A}$ Dashboard foi clara e compreensiva", $33,3 \%$ concordaram, enquanto $66,7 \%$ dos respondentes concordam totalmente. Respondendo ao questionamento sobre "foi fácil tornar-me habilidoso usando o VLA Dashboard", 66,7\% dos respondentes registraram que concordaram, enquanto $33,3 \%$ concordaram totalmente. Do mesmo modo para a questão "é fácil de lembrar como realizar tarefas usando o VLA Dashboard", 66,7\% dos respondentes concordam, enquanto 33,3\% concordaram totalmente. No que se refere à questão "eu achei o VLA Dashboard fácil de usar", 33,3\% concordaram, enquanto 66,7\% dos respondentes concordam totalmente. Assim, com os resultados obtidos do construto de Facilidade de Uso Percebida, é possível constatar que o VLA Dashboard foi avaliado de forma satisfatória pela maioria dos participantes, não havendo registro de discordância ou indiferença quanto à compreensão e facilidade de uso para o mecanismo.

\subsection{Compatibilidade}

Em relação às respostas referentes à Compatibilidade, sobre ao questionamento "o $\boldsymbol{V} \boldsymbol{L A}$ Dashboard é compatível com a maneira como eu trabalho", 66,7\% dos respondentes concordaram, enquanto 33,3\% concordaram totalmente. Em relação as respostas para à questão "usar o VLA Dashboard é compatível com todos os aspectos do meu trabalho", $33,3 \%$ foram indiferentes, enquanto $66,7 \%$ dos respondentes concordam. Sobre a questão "o VLA Dashboard é compatível com a maneira como organizo meu trabalho", $33,3 \%$ foram indiferentes, enquanto $66,7 \%$ dos respondentes concordam totalmente. Respondendo o questionamento sobre “o VLA Dashboard é compatível com a maneira como organizo meu trabalho", $100 \%$ dos respondentes registraram que concordaram.

\subsection{Intenções de Uso Futuro e Comentários dos Professores}

Em referência às respostas relacionadas à Intenção de Uso Futuro da questão "assumindo que o VLA Dashboard estivesse disponível no seu trabalho, eu usaria com regularidade", 33,3\% foram indiferentes, 33,3\% concordam e 33,3\% dos respondentes concordaram totalmente. Sobre a questão "eu preferiria usar o VLA Dashboard a utilizar alternativas como relatório", $33,3 \%$ concordaram, enquanto $66,7 \%$ dos respondentes concordam totalmente. No entanto, em relação às respostas para à questão "eu gostaria de usar o VLA Dashboard no futuro para ajudar no meu trabalho", 33,3\% dos respondentes concordam enquanto $66,7 \%$ concordaram totalmente.

Logo, com o objetivo de registrar as opiniões dos professores sobre outras contribuições do mecanismo, foi incluída ao questionário TAM a pergunta "com base em sua experiência, quais são as vantagens e desvantagens de usar o VLA Dashboard". Em vista disso, temos alguns comentários que foram apontados:

Professor-1: "a desvantagem, no momento inicial, seria a alimentação das questões no banco de questões, sendo que, posteriormente, esse fato seria uma vantagem, pois com o passar de pouco tempo teríamos uma boa quantidade de questões para serem trabalhadas. Como principal vantagem, na minha singela opinião, eu vejo os relatórios apresentados pelo fato de nos ajudarem a ter um diagnóstico do desempenho dos alunos".

Professor-2: “entre as vantagens destaco que: é uma forma interessante de 
avaliação inserindo os alunos nesse tipo de tecnologia; a correção automática faz com que ganhe um tempinho para preparar melhor uma aula, por exemplo; permite melhor identificar quais conteúdos necessitam mais atenção; possibilita uma visualização rápida e didática dos resultados obtidos".

Professor-3: "ter uma ferramenta atrativa para os alunos; Resultados das avaliações imediatamente após a aplicação; apresentação de rendimento da turma;". De acordo com a opinião dos professores pode-se identificar que o VLA Dashboard apresentou vantagens na correção automática das avaliações, apoio na análise de desempenho dos estudantes e da turma, ganho de tempo para dedicar a outras atividades. Também permitiu melhor identificação em conteúdos de necessitam de maior atenção, além de possibilidade de visualização rápida dos resultados obtidas nas avaliações.

\section{Considerações Finais}

Este artigo apresentou o VLA Dashboard, que teve como objetivo principal descrever as contribuições de uso do mecanismo na prática avaliativa do professor de escola pública. Para validação da abordagem, realizou-se um Estudo de Caso com professores de Matemática do $1^{\circ}$ Ano do Ensino Médio de um IFE e validou-se pelo modelo TAM. De acordo com o registro da opinião dos usuários, os resultados apontam que a abordagem é válida, pois amplia as possibilidades de aplicação de intervenções pedagógicas tanto individuais como coletivas com base nas informações fornecidas pelo mecanismo.

Por fim, em relação ao construto Utilidade Percebida, identificou-se que a maioria dos usuários concorda que o VLA Dashboard foi útil e que irá contribuir para melhorar o seu desempenho no trabalho, não havendo discordância sobre a sua utilidade por nenhum de seus usuários. Com relação ao construto Facilidade de Uso Percebida, constatou-se que o VLA Dashboard foi avaliado de forma satisfatória pela maioria dos usuários, não havendo registro de discordância ou indiferença quanto a sua compreensão e facilidade de uso. Isso mostra que os usuários relataram que o uso do Dashboard não demandará esforço desnecessário.

De acordo com os professores, o mecanismo é compatível com o seu modo de trabalho. Isso revela que está dentro do apresentado em sua proposta de contribuir para a prática avaliativa do professor. $\mathrm{O}$ presente trabalho foi focado em Matemática, entretanto, a mesma abordagem pode ser adaptada com pequenos ajustes para outras áreas do conhecimento ministradas no Ensino Médio.

\section{Agradecimentos}

Os autores agradecem o apoio financeiro da Fundação de Amparo à Pesquisa do Estado do Amazonas (FAPEAM) por meio do Programa de Apoio à Formação de Recursos Humanos Pós-Graduados para o Interior do Estado do Amazonas - RH Interiorização Fluxo Continuo - III Chamada, do Edital N003/2015.

\section{Referências}

ARANTES, H.; SEABRA, R. TME: Aplicativo M-Learning para o Estudo de Conceitos Matemáticos com Ênfase no ENEM. In: XXVII Simpósio Brasileiro de Informática na Educação (SBIE), v. 27, n. 1, p. 1-10, 2016.

BELlifEMINE, F.; CAIRE, G.; GREENWOOD, D. Developing Multi-Agent Systems With JADE. England: John Wiley \& Sons Ltd, 2007.

BRASIL. Plano de Desenvolvimento da Educação (PDE): Prova Brasil. BrasíliaDF.: MEC, SEB; INEP, 2008. 
CARD, S., MACKINLAY, J.; SHNEIDERMAN, B. Readings in Information Visualization: Using Vision to Think. San Francisco, CA: Morgan Kaufmann, 1999. CHARLEER, S., KLERKX, J.; DUVAL, E. Learning Dashboards". Journal of Learning Analytics, v. 1, n. 3, p. 199-202, 2014.

CHEN, C. Information Visualization. Wiley Interdisciplinary Reviews: Computational Statistics, v. 2, n. 4, p. 387-403, 2010.

DAVIS, F. Perceived Usefulness, Perceived East of Use, and User Acceptance of Information Technology. MIS Quarterly, v. 13, n. 3, p. 319-340, 1989.

DUVAL, E. Attention Please! Learning Analytics for Visualization and Recommendation. In: LAK '11 Proceedings of the 1st International Conference on Learning Analytics and Knowledge, p. 9-17, 2011.

EINHARDT, L., TAVARES, T. A.; CECHINEL, C. Moodle Analytics Dashboard: A Learning Analytics Tool to Visualize Users Interactions in Moodle. In: Learning Objects and Technology (LACLO), Latin American Conference on, p. 1-6, 2016.

FEW, S. Dashboard Confusion. Perceptual Edge, p. 1-4, 2004.

FEW, S. Information Dashboard Design The Effective Visual Communication of Data. $1^{\text {a }}$ ed. Gravenstein Highway: O'Reilly, 2006.

FISHBEIN, M.; AJZEN, I. Belief. Attitude, Intention and Behavior: An Introduction to Theory and Research. Addison-Wesley, 1975.

GIL, A. C. Como Elaborar Projetos de Pesquisa. $5^{\text {a }}$ ed. São Paulo: Editora Atlas, 2010.

INEP. Sistema de Avaliação da Educação Básica (SAEB). 2015. ed. Brasília-DF., p. 1-49, 2016.

JAQUES, P. A.; OLIVEIRA, F. M. Um Experimento com Agentes de Software para Monitorar a Colaboração em Uma Turma Virtual. In: Revista Brasileira de Informática na Educação (RBIE). n.07, 2000.

JOHNSON, L.; ADAMS, S.; CUMMINS, M. NMC Horizon Report: 2012 Higher Education Edition. Austin, Texas: The New Media Consortium, 2012.

JOHNSON, L. et al. NMC Horizon Report: 2013 Higher Education Edition. Austin, Texas: The New Media Consortium, 2013.

KAMIN, S. N.; CAPITANU, B. A Teacher's Dashboard for High School Algebra Class. USA, p. 1-5, 2008.

MARINHO, E. C. et al. A Brazilian Initiative Towards Digital Literacy: Assessing Motivational Factors of e-Proinfo Using TAM. In: Simpósio Brasileiro de Informática na Educação (SBIE), v. 25, n. 1, p. 1-10, 2014.

PARK, Y.; JO, I. Development of the Learning Analytics Dashboard to Support Students ' Learning Performance. Journal of Universal Computer Science, v. 21, n. 1, p. 110$133,2015$.

PODGORELEC, V.; KUHAR, S. Taking Advantage of Education Data: Advanced Data Analysis and Reporting in Virtual Learning Environments. Elektronika ir Elektrotechnika, v. 114, n. 8, p. 111-116, 2011.

RUSSELL, S.; NORVIG, P. Inteligência Artificial. 3. ed. Rio de Janeiro: Elsevier Brasil, 2013.

SCHWENDIMANN, B.; RODRIGUEZ-TRIANA, M.; VOZNIUK, A. Perceiving Learning at a Glance: A Systematic Literature Review of Learning Dashboard Research. IEEE Transactions on Learning Technologies, n. 1, p. 30-41, 2016.

SIEMENS, G. (2011). 1st International Conference on Learning Analytics and Knowledge 2011 (LAK'11). < https://tekri.athabascau.ca/analytics/>. Acessado em: 27 Mar. 2016.

WOOLDRIDGE, M. An Introduction to Multi-Agent Systems. 2nd. ed. Great Britain: John Wiley \& Sons LTD, 2009.

YIN, R. K. Estudo de Caso: Planejamento e Métodos. 5 a ed. Porto Alegre, MG: Bookman, 2015. 